\title{
On the $\alpha$-migrativity of t-norms and t-conorms over nullnorms and uninorms
}

\author{
Emel Asici \\ Department of Software Engineering, Faculty of Technology, Karadeniz Technical University, 61830 Trabzon, Turkey
}

Received: 24 March 2017, Accepted: 28 April 2017

Published online: 3 March 2018.

\begin{abstract}
In this paper the notions of $\alpha$-migrative triangular norms over a fixed nullnorm and a fixed uninorm are introduced and studied. All solutions of the migrativity equation for all possible combinations of uninorms and nullnorms are analyzed and characterized. Similar study is done for triangular conorms.
\end{abstract}

Keywords: Nulllnorm, uninorm, Migrativity property.

\section{Introduction}

Definition 1. [9] Let $\alpha \in] 0,1\left[\right.$ be given. A binary operation $T:[0,1]^{2} \rightarrow[0,1]$ is said to be $\alpha$-migrative if we have $T(\alpha x, y)=T(x, \alpha y)$ for all $x, y \in[0,1]$.

Clearly the product t-norm $T_{P}$ is $\alpha$-migrative for any $\left.x \in\right] 0,1[$. Many authors investigated $\alpha$-migrative property. The migrativity property has been studied for t-norm in [13,14,15,23], for t-subnorms in [25], for semicopulas, quasi-copulas and copulas in $[10,11,12,22]$.

In [21], it was introduced the definition of $\left(\alpha, U_{0}\right)$-migrative uninorm analyzing some properties. $\left(\alpha, U_{0}\right)$-migrative uninorms were characterized when $U_{0}$ lies in one of the following classes of uninorms: $\mathscr{U}_{\min }$ or $\mathscr{U}_{\text {max }}$, idempotent uninorms, representable uninorms. In [26], it was discussed and characterized the migrative property for the nullnorms. In [20], it was introduced the definition of $(\alpha, T)$-migrative uninorm for a given t-norm $T$, analysing some of its initial properties. The authors continue with the characterization of those $(\alpha, T)$-migrative uninorms, that lay in each one of the most usual classes of uninorms, i.e., uninorms in $\mathscr{U}_{\min }$ and $\mathscr{U}_{\max }$, idempotent uninorms, representable uninorms and uninorms continuous in the open square $] 0,1\left[^{2}\right.$. In [24], it was studied $\alpha$-migrative uninorms over a fixed uninorm, where those two uninorms have different neutral elements. All cases when both uninorm lay in any one of the most usual classes of uninorms are analyzed, characterizing all solutions of the migrativity equation for some possible combinations. Nullnorms, uninorms and t-norms were also studied by many other authors $[1,2,3,4,6,7,8,17,18]$. In the present paper, we introduce the migrativity of triangular norms over nullnorms and over uninorms. The paper is organized as follows. We shortly recall some basic notions in Section 2. In section 3, we introduce the definition of $(\alpha, F)$-migrative triangular norm for a given nullnorm $F$. Also we introduce the definition of $(\alpha, U)$-migrative triangular norm for a given nullnorm $U$. Similar study is done for triangular conorms. That is, we introduce the migrativity of triangular conorms over nullnorms and over uninorms. 


\section{Notations, definitions and a review of previous results}

Definition 2. [18] A triangular norm (t-norm for short) is a binary operation $T$ on the unit interval [0,1], i.e., a function $T:[0,1]^{2} \rightarrow[0,1]$, such that for all $x, y, z \in[0,1]$ the following four axioms are satisfied:

(T1) $T(x, y)=T(y, x), \quad$ (commutativity)

(T2) $T(x, T(y, z))=T(T(x, y), z), \quad$ (associativity)

(T3) $T(x, y) \leq T(x, z)$ whenever $y \leq z, \quad$ (monotonicity)

(T4) $T(x, 1)=x, \quad$ (boundary condition.)

Example 1.[18] The following are the four basic t-norms $T_{M}, T_{P}, T_{L}$ and $T_{D}$ on $[0,1]$ given by, respectively,

$T_{M}(x, y)=\min (x, y)$,

$T_{P}(x, y)=x y$,

$T_{L}(x, y)=\max (x+y-1,0)$,

$T_{D}(x, y)= \begin{cases}0, & \text { if }(x, y) \in\left[0,1\left[^{2}\right.\right. \\ \min (x, y), & \text { otherwise. }\end{cases}$

Definition 3. [18] A triangular conorm (t-conorm for short) is a binary operation $S$ on the unit interval [0,1], i.e., a function $S:[0,1]^{2} \rightarrow[0,1]$ if it is commutative, associative, increasing with respect to the both variables and has a neutral element 0 .

Example 2. [18] The following are the four basic t-conorms $S_{M}, S_{P}, S_{L}$ and $S_{D}$ on [0,1] given by, respectively,

$S_{M}(x, y)=\max (x, y)$,

$S_{P}(x, y)=x+y-x y$

$S_{L}(x, y)=\min (x+y, 1)$

$S_{D}(x, y)= \begin{cases}1, & \text { if }(x, y) \in] 0,1]^{2} \\ \max (x, y), & \text { otherwise. }\end{cases}$

Definition 4. [16] A binary function $U:[0,1]^{2} \rightarrow[0,1]$ is called a uninorm if it is associative, commutative, non-decreasing in each variable and there is a neutral element $e \in[0,1]$ such that $U(e, x)=x$ for all $x \in[0,1]$.

The set $A(e)$ is defined as follows:

$$
A(e)=] 0, e] \times[e, 1[\cup[e, 1[\times] 0, e] .
$$

We denote by $U(e)$ the set of all uninorms on $[0,1]$ with the neutral element $e \in[0,1]$.

Theorem 1. [16] Let $U:[0,1]^{2} \rightarrow[0,1]$ be a uninorm with neutral element $\left.e \in\right] 0,1[$. Then the sections $x \mapsto U(x, 1)$ and $x \mapsto U(x, 0)$ are continuous in each point except perhaps for $e$ if and only if $U$ is given by one of the following formulas.

(a) If $U(0,1)=0$, then

$$
U(x, y)= \begin{cases}e T\left(\frac{x}{e}, \frac{y}{e}\right), & (x, y) \in[0, e]^{2} \\ e+(1-e) S\left(\frac{x-e}{1-e}, \frac{y-e}{1-e}\right), & (x, y) \in[e, 1]^{2} \\ \min (x, y), & (x, y) \in A(e) .\end{cases}
$$

where $T$ is a t-norm and $S$ is a t-conorm.

(b) If $U(0,1)=1$, then the same structure holds, changing minimum by maximum in $A(e)$.

The set of uninorms as in case (a) will be denoted by $\mathscr{U}_{\min }$ and the set of uninorms as in case (b) by $\mathscr{U}_{\text {max }}$. We will denote a uninorm $U$ in $\mathscr{U}_{\min }$ with underlying t-norm $T$, underlying t-conorm $S$ and neutral element $e$ by $U \equiv\langle T, e, S\rangle_{\min }$ and in a similar way, a uninorm in $\mathscr{U}_{\max }$ by $U \equiv\langle T, e, S\rangle_{\max }$. 
Definition 5. [5] A function $F:[0,1]^{2} \rightarrow[0,1]$ is called nullnorm if it is commutative, associative, non-decreasing in each variable and there exists $k \in[0,1]$ called absorbing element that verifies $F(k, x)=k$ for all $x \in[0,1]$ and

$$
F(0, x)=x \text { for all } x \leq k \text { and } F(1, x)=x \text { for all } x \geq k
$$

In that case, when $k=0$ we obtain a t-norm and when $k=1$ we obtain a t-conorm. In general, the absorbing element is always given by $k=F(1,0)$. The structure of nullnorms is given as follows.

Theorem 2. [19] Let $F:[0,1]^{2} \rightarrow[0,1]$ be a nullnorm with absorbing element $F(1,0)=k \notin\{0,1\}$. Then

$$
F(x, y)= \begin{cases}k S\left(\frac{x}{k}, \frac{y}{k}\right), & (x, y) \in[0, k]^{2} \\ k+(1-k) T\left(\frac{x-k}{1-k}, \frac{y-k}{1-k}\right), & (x, y) \in[k, 1]^{2} \\ k, & \text { otherwise }\end{cases}
$$

where $S$ is a t-conorm and $T$ is a t-norm.

A nullnorm $F$ with absorbing element $k$, underlying t-norm $T$ will be denoted by $F \equiv\langle S, k, T\rangle$.

\section{Migrativity of t-norms over nullnorms and uninorms}

Now, we will introduce the definition of migrativity of a t-norm $T$ over a nullnorm $F$.

Definition 6. Given a nullnorm $F$ and $\alpha \in] 0,1[$, a $t$-norm $T$ is called $\alpha$-migrative over $F$ or $(\alpha, F)$-migrative if

$$
T(F(\alpha, x), y)=T(x, F(\alpha, y)) \text { for all } x, y \in[0,1]
$$

Since the extreme values of $a$ correspond to the well known cases of t-norms and t-conorms, we will only deal with nullnorms with absorbing element $a \in] 0,1[$.

Lemma 1. Consider $\alpha \in] 0,1[$. Let $T$ be a $t$-norm, $F$ be a nullnorm with absorbing element $a$. Then $T$ is not $\alpha$-migrative over $F$.

Proof. (i) Let $\alpha=a$. If $T$ is $\alpha$-migrative over $F$, then

$$
T(F(\alpha, 1), 0)=T(\alpha, 0)=0<\alpha=T(1, \alpha)=T(1, F(\alpha, 0))
$$

It leads a contradiction that $T$ is not $\alpha$-migrative over $F$ for $\alpha=a$.

(ii) Let $\alpha \in] 0, a[$. If $T$ is $\alpha$-migrative over $F$, then

$$
T(0, F(\alpha, 1))=0<\alpha=T(\alpha, 1)=T(F(\alpha, 0), 1)
$$

It leads a contradiction that $T$ is not $\alpha$-migrative over $F$ for $\alpha \in] 0, a[$.

(iii) Let $\alpha \in] a, 1[$. If $T$ is $\alpha$-migrative over $F$, then

$$
T(F(\alpha, 1), 0)=0<a<F(\alpha, 0)=T(1, F(\alpha, 0)) .
$$

It leads a contradiction that $T$ is not $\alpha$-migrative over $F$ for $\alpha \in] a, 1[$. 
Now, we will introduce the definition of migrativity of a t-conorm $S$ over a nullnorm $F$.

Definition 7. Given a nullnorm $F$ and $\alpha \in] 0,1[$, a t-conorm $S$ is called $\alpha$-migrative over $F$ or $(\alpha, F)$-migrative if

$$
S(F(\alpha, x), y)=S(x, F(\alpha, y)) \text { for all } x, y \in[0,1]
$$

Since the extreme values of $a$ correspond to the well known cases of t-norms and t-conorms, we will only deal with nullnorms with absorbing element $a \in] 0,1[$.

Lemma 2. Consider $\alpha \in] 0,1[$. Let $S$ be a t-conorm, $F$ be a nullnorm with absorbing element $a$. Then $S$ is not $\alpha$-migrative over $F$.

Proof. (i) Let $\alpha \in[a, 1[$. If $S$ is $\alpha$-migrative over $F$, then

$$
S(F(\alpha, 1), 0)=S(\alpha, 0)=\alpha<1=S(1, F(\alpha, 0)) .
$$

It leads a contradiction that $S$ is not $\alpha$-migrative over $F$.

(ii) Let $\alpha \in] 0, a[$. If $S$ is $\alpha$-migrative over $F$, then

$$
S(0, F(\alpha, 1))=F(\alpha, 1)<a<1=S(F(\alpha, 0), 1) .
$$

It leads a contradiction that $S$ is not $\alpha$-migrative over $F$.

Similarly to the case of nullnorms we want to study the migrativity of t-norms over uninorms.

Definition 8. Given a uninorm $U$ and $\alpha \in] 0,1[$, a t-norm $T$ is called $\alpha$-migrative over $U$ or $(\alpha, U)$-migrative if

$$
T(U(\alpha, x), y)=T(x, U(\alpha, y)) \text { for all } x, y \in[0,1] \text {. }
$$

Since the extreme values of $e$ correspond to the well known cases of t-norms and t-conorms, we will only deal with uninorms with neutral element $e \in] 0,1[$.

Lemma 3. Consider $T$ be a t-norm and $U$ be a uninorm with neutral element e. Then, $T$ is e-migrative over $U$.

Proof. $T(U(e, x), y)=T(x, y)=T(x, U(e, y))$ for all $x, y \in[0,1]$

Lemma 4. Consider $\alpha \in] 0, e[$. Let $T$ be a t-norm, $U$ be a uninorm with neutral element e. Then $T$ is not $\alpha$-migrative over $U$.

Proof. Since $0<\alpha$, we have that $U(0,0)=0<U(\alpha, 0)$. If $T$ is $\alpha$-migrative over $U$, then we have

$$
T(0, U(\alpha, 1))=0<U(\alpha, 0)=T(U(\alpha, 0), 1)
$$

contradiction. So, $T$ is not $\alpha$-migrative over $U$.

Lemma 5. Consider $\alpha \in]$ e, $1[$. Let $T$ be a $t$-norm, $U$ be a uninorm with neutral element e. Then $T$ is not $\alpha$-migrative over $U$.

Proof. Since $e<\alpha$, we have

$$
1=U(e, 1)<U(\alpha, 1)
$$


by the monotonicity of $U$. So, it is obtained that $U(\alpha, 1)=1$. If $T$ is $\alpha$-migrative over $U$, then we have

$$
T(e, U(\alpha, 1))=T(e, 1)=e<\alpha=T(\alpha, 1)=T(U(\alpha, e), 1)
$$

contradiction. So, $T$ is not $\alpha$-migrative over $U$.

Definition 9. Given a uninorm $U$ and $\alpha \in] 0,1$ [, a t-conorm $S$ is called $\alpha$-migrative over $U$ or $(\alpha, U)$-migrative if

$$
S(U(\alpha, x), y)=S(x, U(\alpha, y)) \text { for all } x, y \in[0,1]
$$

Since the extreme values of $e$ correspond to the well known cases of t-norms and t-conorms, we will only deal with uninorms with neutral element $e \in] 0,1[$.

Lemma 6. Consider $S$ be a t-conorm and $U$ be a uninorm with neutral element e. Then, $S$ is e-migrative over $U$.

Proof. $S(U(e, x), y)=S(x, y)=S(x, U(e, y))$ for all $x, y \in[0,1]$.

Lemma 7. Consider $\alpha \in] 0, e[$. Let $S$ be a t-conorm, $U$ be a uninorm with neutral element . Then $S$ is not $\alpha$-migrative over $U$.

Proof. Since $\alpha<e$, we have that

$$
U(\alpha, 0)<U(e, 0)=0
$$

by the monotonicity of $U$. So, it is obtained that $U(\alpha, 0)=0$. If $S$ is $\alpha$-migrative over $U$, then we have

$$
S(U(\alpha, e), 0)=U(\alpha, e)=\alpha<e=S(e, 0)=S(e, U(\alpha, 0))
$$

contradiction. So, $S$ is not $\alpha$-migrative over $U$.

Lemma 8. Consider $\alpha \in]$ e, $1[$. Let $S$ be a t-conorm, $U$ be a uninorm with neutral element e. Then $S$ is not $\alpha$-migrative over $U$.

Proof. Since $e<\alpha$, it is obtained that $1=U(e, 1)<U(\alpha, 1)$. If $S$ is $\alpha$-migrative over $U$, then we have a

$$
S(U(\alpha, 1), 0)=U(\alpha, 1)=1=S(1, U(\alpha, 0))
$$

contradiction. So, $S$ is not $\alpha$-migrative over $U$.

\section{Conclusions}

We have introduced and studied the migrativity of t-norms over nullnorms and the migrativity of t-norms over uninorms. Similar definition is done for t-conorms.

\section{Competing interests}

The authors declare that they have no competing interests.

\section{Authors' contributions}

All authors have contributed to all parts of the article. All authors read and approved the final manuscript. 


\section{References}

[1] E. Aşıc1, An order obtained from nullnorms and its properties, Fuzzy Sets Syst. 325 (2017) 35-46.

[2] E. Aşıc1, On the properties of the F-partial order and the equivalence of nullnorms, Fuzzy Sets Syst. doi: 10.1016/j.fss.2017.11.008, in press.

[3] E. Aşıcı and F. Karaçal, Incomparability with respect to T-partial order, Kybernetika. 52 (2016) 15-27.

[4] G. Birkhoff, Lattice Theory, 3 rd edition. Providence 1967.

[5] T. Calvo, B.De Baets and J.Fodor, The functional equations of Frank and Alsina for uninorms and nullnorms, Fuzzy Sets Syst. 120 (2001) 385-394.

[6] B. De Baets and R. Mesiar, Triangular norms on the real unit square, Proceedings of the 1999 EUSFLAT-ESTYLF Joint Conference, Palma de Mallorca, Spain, 1999, 351-354.

[7] G.D. Çaylı, F. Karaçal, R.Mesiar, On a new class of uninorms on bounded lattices, Inf. Sci. 367-368 (2016) 221-231.

[8] P. Drygas, Distributivity between semi-t-operators and semi-nullnorms, Fuzzy Sets Syst. 264 (2015) 100-109.

[9] F.Durante and P.Sarkoci, A note on the convex combinations of triangular norms, Fuzzy Sets Syst. 159 (2008) 77-80.

[10] F.Durante, J.Fernández-Sánchez and J.J.Quesada-Molina, On the $\alpha$-migrativity of multivariate semi-copulas, Inf. Sci. 187 (2012) 216-223.

[11] F.Durante and R.G.Ricci, Supermigrative semi-copulas and triangular norms, Inf. Sci. 179 (2009) 2689-2694.

[12] J.Fernández-Sánchez, J.J.Quesada-Molina and M.Úbeda-Flores, On ( $\alpha, \beta)$-homogeneous copulas, Inf.Sci. 221 (2013) 181-191.

[13] J.Fodor and I.J.Rudas, An extension of the migrative property for triangular norms, Fuzzy Sets Syst. 168 (2011) 70-80.

[14] J.Fodor and I.J.Rudas, On continuous triangular norms that are migrative, Fuzzy Sets Syst. 158 (2007) 1692-1697.

[15] J.Fodor and I.J.Rudas, Migrative t-norms with respect to continuous ordinal sums, Inf. Sci. 181 (2011) 4860-4866.

[16] J.Fodor, I.J.Rudas and A. Rybalov, Structure of uninorms, Int. J. Uncertain. Fuzziness Knowl.-Based Syst. 5 (1997) $411-427$.

[17] F. Karaçal and E. Aşıcı, Some notes on T-partial order, Journal of Inequalities and Applications. (2013) 219.

[18] E.P. Klement, R. Mesiar and E. Pap, Triangular Norms, Kluwer Academic Publishers, Dordrecht 2000.

[19] M.Mas, G.Mayor and J.Torrens, t-Operators, Int. J. Uncertainty Fuzz. Knowledge-Based Syst. 7 (1999) 31-50.

[20] M.Mas, M.Monserrat, D.Ruiz-Aquilera and J.Torrens, Migrative uninorms and nullnorms over t-norms and t-conorms, Fuzzy Sets Syst. 261 (2015) 20-32.

[21] M.Mas, M.Monserrat, D.Ruiz-Aquilera and J.Torrens, An extension of the migrative property for uninorms, Fuzzy Sets Syst. 246 (2013) 191-198.

[22] R.Mesiar, H.Bustince and J.Fernandez, On the $\alpha$-migrativity of semicopulas, quasi-copulas and copulas, Inf.Sci. 180 (2010) $1967-$ 1976.

[23] Y.Ouyang, Generalizing the migrativity of continuous t-norms, Fuzzy Sets Syst. 211 (2013) 73-83.

[24] Y.Su, W.Zong and H.W Liu, Migrative property for uninorms, Fuzzy Sets Syst. 5 (2014) 749-759.

[25] L.Wu, Y.Ouyang, On the migrativity of triangular subnorms, Fuzzy Sets Syst. 226 (2013) 89-98.

[26] W.Zong, Y.Su and H.W Liu, Migrative property for nullnorms, Int. J. Uncertainty Fuzz. Knowledge-Based Syst. 5 (2014) $749-759$. 